\title{
A atuação do enfermeiro em unidade de terapia intensiva na pandemia de COVID-19: relato de experiência
}

\author{
Nurses' performance in an intensive care unit in the COVID-19 pandemic: experience \\ report
}

Desempeño de enfermeras en una unidad de cuidados intensivos en la pandemia

COVID-19: informe de experiência

Maurício Rouvel Nunes ${ }^{1 *}$.

\begin{abstract}
RESUMO
Objetivo: Relatar a atuação do enfermeiro de uma unidade de terapia intensiva (UTI) adulto de pacientes com COVID-19 em hospital público de referência no Sul do país. Relato de experiencia: Trata-se de um estudo descritivo, narrativo, do tipo relato de experiência, referente a atuação do enfermeiro em uma unidade de terapia intensiva na pandemia de COVID-19 em um hospital público de referência, em Porto Alegre, RSBrasil no período de maio a agosto de 2020. Na assistência de enfermagem ao paciente crítico com COVID19 deparou-se com uma nova realidade de cuidado nos serviços de enfermagem em terapia intensiva. A admissão dos pacientes em UTI requer a utilização de uma gama de intervenções técnico-científicas, diante da instabilidade fisiológica e dos riscos à saúde apresentados. Frente a este cenário, se faz necessário conhecer tecnologias e conhecimento acerca do atendimento aos pacientes com COVID-19 com o intuito de pautar a nossa assistência em um cuidado baseado em evidências. Considerações finais: A pandemia de COVID-19 trouxe enormes desafios para as equipes de enfermagem no que concerne a mudanças de práticas assistenciais e readequações na gestão de trabalho. Portanto, é necessário instrumentalizar os profissionais com conhecimentos sobre novo coronavírus e propiciar ambientes de trabalho adequados.
\end{abstract}

Palavras-chave: Cuidados de enfermagem, Unidades de terapia intensiva, Infecções por coronavírus.

\begin{abstract}
Objective: To report the role of nurses in an adult intensive care unit (ICU) for patients with COVID-19 in a public referral hospital in the south of the country. Experience report: This is a descriptive, narrative, experience report type study, referring to the nurse's performance in intensive care unit in the COVID-19 pandemic in a public reference hospital in Porto Alegre, RS-Brazil in May to August 2020. In nursing care for critically ill patients with COVID-19, we are faced with a new reality of care in intensive care nursing services. The admission of patients to the ICU requires the use of a range of technical-scientific interventions, given the physiological instability and health risks presented. Faced with this scenario, it is necessary to know technologies and knowledge about the care to patients with COVID-19 in order to guide our assistance in evidence-based care. Final considerations: The COVID-19 pandemic brought enormous challenges for nursing teams in terms of changes in care practices and readjustments in work management. Therefore, it is necessary to equip professionals with knowledge about the new coronavirus and provide adequate work environments.
\end{abstract}

Keywords: Nursing care, Intensive care units, Coronavirus infections.

${ }^{1}$ Universidade Federal de Ciências da Saúde de Porto Alegre, Porto Alegre - RS.

*E-mail:mmrouvelnunes@gmail.com 


\section{RESUMEN}

Objetivo: Informar el rol del enfermero en una unidad de cuidados intensivos (UCl) de adultos para pacientes con COVID-19 en un hospital público de referencia del sur del país. Informe de experiencia: Se trata de un estudio descriptivo, narrativo, tipo relato de experiencia, referido al desempeño del enfermero en unidade de cuidados intensivos en la pandemia de COVID-19 en un hospital público de referencia en Porto Alegre, RSBrasil en mayo a agosto de 2020. En la atención de enfermería al paciente crítico con COVID-19, nos enfrentamos a una nueva realidad de la atención en los servicios de enfermería de cuidados intensivos. El ingreso de pacientes en $\mathrm{UCl}$ requiere el uso de una serie de intervenciones técnico-científicas, dada la inestabilidad fisiológica y los riesgos para la salud que presenta. Ante este escenario, es necesario conocer tecnologías y conocimientos sobre la atención a los pacientes con COVID-19 para orientar nuestra asistencia en la atención basada en la evidencia. Consideraciones finales: La pandemia de COVID-19 ha traído enormes desafíos para los equipos de enfermería en cuanto a cambios en las prácticas asistenciales y reajustes en la gestión del trabajo. Por tanto, es necesario dotar a los profesionales de conocimientos sobre el nuevo coronavirus y proporcionar entornos laborales adecuados.

Palabras clave: Atención de enfermería, Unidades de cuidados intensivos, Infecciones por coronavirus.

\section{INTRODUÇÃO}

A doença respiratória aguda causada pelo novo coronavírus, COVID-19, que primeiramente se disseminou pela China e posteriormente para outros países, foi declarada no início do ano uma emergência de saúde pública pela Organização Mundial de Saúde (OMS). Os sinais clínicos da doença incluem febre, tosse, fadiga, sintomas gastrointestinais e dificuldade respiratória (GUO YR, et al., 2020).

Desde a sua descoberta, muitos pacientes com a infeção do novo coronavírus (SARS-CoV-2) desenvolveram a síndrome respiratória aguda grave e necessitaram de internação em unidades de terapia intensiva (UTI). Assim, a pandemia se mostrou como um grande estressor dos sistemas de saúde mundiais devido à grande demanda por leitos de UTI e por profissionais de enfermagem (RAURELL-TORREDÀ M, 2020).

No Brasil, as desigualdades sociais e de saúde foram fundamentais para que determinadas regiões no país, devido à escassez de leitos de UTI e de recursos humanos, tivessem taxas de mortalidade superiores à média nacional. No SUS, mais de $72 \%$ das regiões tinham número de leitos inferior ao recomendado pelos órgãos sanitários, levando a superlotação ao desgaste dos respectivos sistemas de saúde. A disseminação espacial do novo coronavírus no país está diretamente associado a cobertura da rede de atenção à saúde bem como ao arsenal médico assistencial presente nessas regiões (RACHE B, et al., 2020).

Em pesquisas realizadas em UTIs, encontrou-se uma taxa de mortalidade de pacientes com COVID-19 de ao redor de $50 \%$ e mais de $75 \%$ necessitaram de ventilação mecânica invasiva (VMI) com um tempo de internação superior a duas semanas, assim, demonstrando a necessidade de uma equipe de enfermeiros e técnicos de enfermagem capacitados para atuação nesses cenários de cuidado, em que o estresse e demanda de trabalho são intensos (RAURELL-TORREDÀ M, 2020; BHATRAJU PK, et al., 2020)

Os pacientes com a infecção causada pelo coronavírus necessitam de uma assistência de enfermagem qualificada, pois o manejo da COVID-19 é desafiador nas UTIs. No que concerne as terapias utilizadas pelos pacientes, salienta que a ventilação mecânica invasiva, a realização da manobra prona e a prevenção e o tratamento de dellirium são pontos desafiadores para a equipe de enfermagem (BHATRAJU PK, et al., 2020)

Diante deste cenário pandêmico, os profissionais enfermeiros encontram desafios importantes no que tange a assistência e qualidade dos serviços de enfermagem em terapia intensiva. As experiências de enfermeiros da China e Itália mostrou que um dos maiores desafios na assistência aos pacientes com COVID19 deu-se do âmbito da organização de equipes treinadas e capacitadas, dimensionamento de pessoal, gerenciamento de insumos e equipamentos e a atenção à saúde mental destes profissionais (KANG L, et al., 2020; SHARMA SK, et al., 2020).

REAS/EJCH | Vol.12(11) | e4935 | DOI: https://doi.org/10.25248/reas.e4935.2020 Página 2 de 6 
Assim, o objetivo do trabalho é relatar a atuação do enfermeiro de uma unidade de terapia intensiva adulto de pacientes com COVID-19 em hospital público de referência no Sul do país. A finalidade é trazer para o âmbito de discussão os desafios da assistência e as práticas de enfermagem ao paciente crítico com COVID19, bem como relatar as demandas de saúde mental dos profissionais na assistência em saúde.

\section{RELATO DE EXPERIÊNCIA}

Trata-se de um estudo descritivo, narrativo, do tipo relato de experiência, referente a atuação do enfermeiro em uma unidade de terapia intensiva na pandemia de COVID-19 em um hospital público de referência, em Porto Alegre, RS-Brasil no período de maio a agosto de 2020. Na assistência de enfermagem ao paciente crítico com COVID-19 deparou-se com uma nova realidade de cuidado nos serviços de enfermagem em terapia intensiva. A admissão dos pacientes em UTI requer uma equipe multiprofissional capacitada para ao atendimento devido às condições críticas, as comorbidades associadas e aos riscos à saúde apresentados durante a internação.

Acrescenta-se isso a rede de suporte social fragilizada que muitos pacientes possuem, dificultando o acolhimento destes usuários ao serviço de saúde. Os desafios no que concerne ao gerenciamento de recursos e materiais são enormes, exigindo dos serviços de saúde e dos gestores estratégias e planos de enfretamento a esta nova realidade. Diante da pandemia do novo coronavírus, percebe-se que a conciliação do fazer técnico com as práticas de humanização nesses espaços, tornam-se um desafio devido à grande demanda exigida aos profissionais de enfermagem. Nesta perspectiva, descrever as experiências no atendimento destes pacientes torna-se importante como forma de discutir as relações terapêuticas estabelecidas entre a equipe de enfermagem e os pacientes críticos.

A as ações voltadas a educação permanente em saúde, são de fundamental importância para melhoria da qualidade no atendimento aos pacientes com o novo coronavírus, haja vista a demanda crescente por profissionais para atuação em UTIs. Além disso, o estabelecimento de canais de diálogo entre as equipes multiprofissionais no âmbito da terapia intensiva, são de fundamental importância no contexto vivenciado. $O$ aumento no fluxo de atendimentos a pacientes com COVID-19 iniciou-se no mês de maio, exigindo do serviço de terapia intensiva do hospital a revisão e padronização do uso correto da paramentação e de equipamentos de proteção individual (EPI). A gravidade clínica apresentada pelos pacientes requereu da equipe de enfermagem grande demanda de trabalho.

$\mathrm{Na}$ admissão, a assistência de enfermagem era pautada na realização de procedimentos de enfermagem, estabilização clínica e no estabelecimento da comunicação com os familiares. No cotidiano do serviço, a equipe deparou-se com agravamento clínico dos pacientes, a internação prolongada e a dificuldade na recuperação deles, oriundo do acometimento sistêmico causado pela doença. Assim, os profissionais vivenciaram a angústia e um sentimento de incapacidade diante o cuidado aos pacientes com COVID-19.

Outro ponto importante a destacar, foi a adoção de reuniões semanais por meio de videoconferência com a finalidade de capacitar os profissionais para a melhoria de práticas assistenciais, pois muitos profissionais ingressantes não tinham experiência no atendimento ao paciente crítico. Possibilitando, assim, uma melhoria na coordenação do cuidado e da comunicação entre os profissionais. Frente a esta demanda elevada nos serviços de saúde, em especial as UTIs, os enfermeiros possuem papel central no enfrentamento a esta emergência em saúde pública.

Esta realidade trouxe em evidência alguns aspectos importantes a enfermagem brasileira, como o quantitativo inadequado de profissionais, baixos salários e condições de trabalho inadequadas. Assim, com o intuito de pautar a nossa assistência em um cuidado baseado em evidências faz-se necessário conhecer tecnologias e conhecimento acerca do atendimento aos pacientes com COVID-19.

\section{DISCUSSÃO}

A pandemia de COVID-19 representa o maior desafio em saúde do século XXI até o presente momento. A doença possui repercussões importantes nos sistemas de saúde nacionais, especificamente no que concerne a criação e manutenção de leitos em terapia intensiva, aquisição de ventiladores mecânicos, organização dos serviços de saúde e pesquisas acerca de vacinas e terapêuticas utilizadas nos pacientes. 
No Brasil, a pandemia exacerba problemas estruturais presentes em nosso sistema de saúde como falta de investimentos em pesquisa, déficit de profissionais qualificados nos serviços de saúde e a desarticulação presente entre os níveis de atenção à saúde (BRITO SBP, et al., 2020). No âmbito do cuidado aos pacientes críticos, depara-se com uma demanda cada vez maior profissionais enfermeiros qualificados que possam atuar nos serviços de terapia intensiva.

Viana RAPP, et al. (2014), em estudo realizado pela Universidade Federal de São Paulo e Universidade Federal de Santa Catarina, evidenciou que os profissionais de UTIs devem se pautar no domínio da linguagem tecnológica específica e na assistência integral e segura.

Os maiores entraves no processo de qualificação dos enfermeiros intensivista são a ausência de programas de educação permanente nas instituições, a falta de tempo para se qualificar, o cansaço e estresse e, também, a dificuldade de conciliar atividades educativas com as demandas de trabalho, dificuldades estas no qual convivíamos e que estão sendo acentuadas pela pandemia.

A prática de enfermagem em terapia intensiva se insere em um contexto em que se tem pacientes com grau elevado de complexidade e elevada dependência de profissionais de enfermagem. Sendo considerado uma prática de atuação com dinâmica própria no processo de cuidar, altamente instrumentalizada e pautada no uso de tecnologias leves, leve-duras e duras de cuidado. Assim, os profissionais estão em constante situações de desgaste físico e mental, tornando-o de fundamental importância a compreensão e melhoria das dinâmicas de trabalho (CAMPOS JF, et al., 2014).

\section{A atuação do enfermeiro e as Tecnologias do cuidado em saúde}

As tecnologias do cuidado em saúde são todas as técnicas, procedimentos e conhecimentos utilizados pelo enfermeiro no cuidado. A discussão acerca da temática de tecnologias de cuidado deve ser pautada na construção de saberes e intervenções no processo saúde-doença que proporcionem o efeito desejado.

As tecnologias envolvidas no trabalho em saúde, podem ser classificadas como leves, que são as tecnologias de produção de vínculo; leve-duras, que são os saberes estruturados que operam no trabalho em saúde e as duras que são as tecnologias, máquinas e equipamentos utilizados no processo de cuidar (OUCHI JD, et al., 2018).

O contexto de assistencial aos pacientes em UTI, muitas vezes é desafiador. Pois, são ambientes om ruídos, alarmes e de procedimentos invasivos constantes, soma-se ao fato de que a estruturação destes ambientes pautados na burocracia e na despersonalização dos pacientes, são limitadores na implementação de políticas voltadas à humanização (OUCHI JD, et al., 2018).

Nos últimos anos, as políticas de visitação das UTIs vinham em um processo de ampliação com a inserção das famílias no cuidado ao paciente, com o intuito de aumentar o engajamento e a satisfação dos familiares e pacientes nos ambientes críticos.

No entanto, a pandemia de coronavírus fez com que muitas destas conquistas realizadas retrocedessem. As medidas restritivas e de bloqueio ao acesso as unidades de cuidado aos pacientes com COVID-19, especificamente, as unidades de terapia intensiva tiveram que ser impostas para proteção de pacientes e familiares.

As informações que anteriormente eram compartilhadas com familiares e pacientes durante as visitas, foram substituídas por chamadas telefônicas e por vídeo como meio de informar as condições clínicas diárias desses pacientes, assim os aparelhos telefônicos acabaram se tornando o último elo afetivo entre os familiares e os pacientes (IMBRIACO G e ALESSANDRO M, 2020). Torna-se crucial a manutenção dos esforços para a humanização nesses ambientes, mesmo em tempos de pandemia.

No que tange aos aspectos clínicos, os pacientes críticos com COVID-19 possuem sintomatologia variada como febre, tosse, dispneia e fadiga. A maioria possui dano pulmonar importante com presença de opacidades bilaterais e consolidação pulmonar nos exames de tomografia computadorizada e radiografia de tórax. Há também alterações de coagulação como aumento do tempo de protrombina (FERNANDES CA, et al., 2020). 
A complicação mais comum nos pacientes é a síndrome da angústia respiratória aguda (SDRA), acrescentando-se outras complicações como injúria renal aguda, lesões cardíacas e infecções secundárias. No que tange a assistência de enfermagem a essas condições clínicas, a utilização de terapia renal substitutiva (TSR) e a utilização da manobra prona tem sido um dos procedimentos mais utilizados nos ambientes críticos como forma de reduzir a mortalidade nestes pacientes com coronavírus (FERNANDES CA, et al., 2020).

No que concerne a manobra prona, o enfermeiro possui um papel fundamental na prática clínica para a organização do processo de implementação da técnica. No Brasil, há vasta literatura acerca da implementação por meio de checklist sobre a manobra prona. A observância de uma padronização nas UTIs é de fundamental importância no atual momento, além disso, cabe salientar que a manobra possui complicações importantes, como: lesões por pressão, pneumonia associada à ventilação mecânica e extubação acidental (OLIVEIRA VM, et al., 2020).

As lesões renais têm sido frequentes nos pacientes com COVID-19, cerca de $40 \%$ dos pacientes têm lesão renal aguda na admissão hospitalar. Destes, $20 \%$ requerem terapia de substituição renal em sua evolução clínica, com uma taxa de mortalidade ao redor de $30 \%$. As causas para esta condição são multifatoriais e frequentemente estão associados a fatores como sepse, hipovolemia e citotóxica do vírus SARS-Cov-2 no epitélio tubular renal.

O manejo de instalação e manutenção das terapias dialíticas são de função dos profissionais enfermeiros com formação adequada, sendo assim, é de fundamental importância a reorganização dos serviços de saúde para o atendimento da demanda crescente de TSR em pacientes com COVID-19 (ARNEDO RD, et al., 2020). Assim, por meio da articulação da equipe multiprofissional no cuidado aos pacientes críticos, pode-se alcançar resultados satisfatórios nos desfechos clínicos.

\section{A saúde mental do profissional enfermeiro em UTI}

O trabalho de enfermagem no cuidado aos pacientes críticos é gerador de sofrimento psíquico. No contexto que vivenciamos, de isolamento social e de pressão nos serviços de saúde, se intensifica os sentimentos de tristeza e abandono e, também, repercussões orgânicas como distúrbio de sono e apetite.

A organização Mundial de Saúde (OMS) alerta desde o início da pandemia que os enfermeiros são os profissionais mais suscetíveis a desgastes físico e mentais intensos, bem como um risco aumentado para o desenvolvimento da Síndrome de Burnout (DE HUMEREZ DC, et al., 2020).

A responsabilidade técnica e a sobrecarga de trabalho do profissional enfermeiro, pode contribuir para o desequilíbrio emocional desses profissionais. Soma-se a esta situação, a pandemia de COVID-19, que resultou em mudanças drásticas nas relações sociais e de trabalho faz com que os profissionais de enfermagem sejam mais suscetíveis ao sofrimento psíquico.

A adoção de estratégias de enfrentamento, o apoio psicológico especializado e a escuta ativa são aspectos importantes a serem debatidos como forma de melhorar as condições de trabalho dos profissionais de enfermagem (DAL BOSCO EB, et al., 2020).

\section{REFERÊNCIAS}

1. ARNEDO RD, et al. Terapias de purificación sanguínea en COVID-19. Revista Colombiana de Nefrología. 2020; 7(2).

2. BHATRAJU PK, et al. Covid-19 in Critically III Patients in the Seattle Region - Case Series. N Engl J Med. 2020; 382(21): 2012-2022.

3. BRITO SBP, et al. Pandemia da COVID-19: o maior desafio do século XXI. Vigilância Sanitária em Debate: Sociedade, Ciência \& Tecnologia. 2020; 8(2): 54-63.

4. CAMPOS JF, et al. Prazer e sofrimento: avaliação de enfermeiros intensivistas à luz da psicodinâmica do trabalho. Escola Anna Nery. 2014; 18(1): 90-95.

5. DAL BOSCO EB, et al. A saúde mental da enfermagem no enfrentamento da COVID-19 em um hospital universitário regional. Rev. Bras. Enferm. 2020; 73(2): e20200434.

6. DE HUMEREZ DC, et al. Saúde mental dos profissionais de enfermagem do brasil no contexto da pandemia covid19: ação do conselho federal de enfermagem. Cogitare Enfermagem. 2020; 25. 
7. FERNANDES CA, et al. Desafios e recomendações para o cuidado intensivo de adultos críticos com doença de coronavírus 2019 (COVID-19). Health Residencies Journal-HRJ. 2020; (1): 21-47.

8. GUO YR, et al. The origin, transmission and clinical therapies on coronavirus disease 2019 (COVID-19) outbreak - an update on the status. Mil Med Res. 2020; 7(1):11.

9. IMBRIACO G, ALESSANDRO M. Names and numbers: How COVID-19 impacted on de-humanization of ICU patients. Journal of the Intensive Care Society. 2020: 1751143720925976.

10. KANG L, et al. Impact on mental health and perceptions of psychological care among medical and nursing staff in Wuhan during the 2019 novel coronavirus disease outbreak: A cross-sectional study. Brain Behav Immun. 2020; 87: 11-17.

11. OLIVEIRA VM, et al. Checklist da prona segura: construção e implementação de uma ferramenta para realização da manobra de prona. Revista Brasileira de Terapia Intensiva. 2017; 29(2): 131-141.

12. OUCHI JD, et al. O papel do enfermeiro na unidade de terapia intensiva diante de novas tecnologias em saúde. Rev Saúde em Foco. 2018; 10: 412-428.

13. RACHE B, et al. Necessidades de infraestrutura do SUS em preparo à COVID-19: leitos de UTI, respiradores e ocupação hospitalar. São Paulo: Instituto de Estudos para Políticas de Saúde (2020).

14. RAURELL-TORREDÀ M. Gestión de los equipos de enfermería de uci durante la pandemia covid-19. Enfermeria Intensiva. 2020; 31(2): 49.

15. SHARMA SK, et al. Clinical nursing care guidance for management of patient with COVID-19. J Pak Med Assoc. 2020; 70(Suppl 3) (5): S118-S123.

16. VIANA RAPP, et al. "Perfil do enfermeiro de terapia intensiva em diferentes regiões do Brasil." Texto \& ContextoEnfermagem. 2014; 23(1): 151-159. 